\title{
A new fluctuation identity for Lévy processes and some applications
}

\author{
LARBI ALILI ${ }^{1}$ and LOÏC CHAUMONT ${ }^{2}$ \\ ${ }^{1}$ Technische Universität Wien, Wiedner Haupstrasse 8-10, A-1040 Vienna, Austria. \\ E-mail: alili@fam.tuwien.ac.at \\ ${ }^{2}$ Laboratoire de Probabilités et Modèles Aléatoires, Tour 56, Université Pierre et Marie Curie, \\ 4 place Jussieu, 75252 Paris Cedex05.E-mail: loc@ccr.jussieu.fr
}

Let $\tau$ and $H$ be respectively the ladder time and ladder height processes associated with a given Lévy process $X$. We give an identity in law between $(\tau, H)$ and $\left(X, H^{*}\right), H^{*}$ being the right-continuous inverse of the process $H$. This allows us to obtain a relationship between the entrance law of $X$ and the entrance law of the excursion measure away from 0 of the reflected process $\left(X_{t}-\right.$ $\left.\inf _{s \leqslant t} X_{s}, t \geqslant 0\right)$. In the stable case, some explicit calculations are provided. These results also lead to an explicit form of the entrance law of the Lévy process conditioned to stay positive.

Keywords: excursion measure; fluctuation theory; Lévy processes; local time

\section{Introduction}

Let $X$ be a real-valued Lévy process starting from 0 such that 0 is regular for both $(-\infty, 0)$ and $(0, \infty)$. The associated past maximum process $\bar{X}$ is defined, for a fixed $t \geqslant 0$, by $\bar{X}_{t}=\sup _{s \leqslant t} X_{s}$. It is well known that the reflected process $\bar{X}-X$ is strongly Markovian. So we can construct its local time at 0 , which we denote by $L$, in the usual sense (see Bertoin 1996, Chapter IV). The right-continuous inverse of $L$, which we denote by $\tau$, is usually called the ladder time process, and the corresponding height $H \stackrel{\text { def }}{=} X_{\tau}$ is called the ladder height process. The ladder processes $(\tau, H)$ and $(\hat{\tau}, \hat{H})$, associated with $X$ and its dual process $\hat{X} \stackrel{\text { def }}{=}-X$, are bivariate subordinators whose respective laws entirely characterize the law of $X$. It is normal practice to express the law of functionals of $X$ in terms of the ladder process' characteristic exponents. Indeed, it is easier to deal with subordinators rather than with the Lévy process itself and that is an important reason for the study of ladder processes.

This paper is organized as follows. Our first purpose is to reinforce the relationships recalled in Section 2 between the law of $X$ and the law of the ladder processes. More precisely, in Section 3, we give a disintegration formula of the identity

$$
\frac{1}{t} P\left(X_{t} \in \mathrm{d} x\right)=\int_{0}^{\infty} \frac{\mathrm{d} u}{u} P\left(\tau_{u} \in \mathrm{d} t, X_{\tau_{u}} \in \mathrm{d} x\right), \quad t>0,
$$

discovered, for continuous time, by Bertoin and Doney (1997). For the stable case, some more explicit relations are stated in Corollaries 2 and 3. Section 4 is devoted to other similar identities, and we provide some simple applications of the fundamental identity. In Section 5 
we show how identity (3.1) can be applied to derive the entrance law of the reflected process's excursion measure away from 0 . In particular, we provide a description of the density between this entrance law and the entrance law of $X$ on $(0, \infty)$. This result leads to an explicit formula for the entrance law of the Lévy process conditioned to stay positive which is closely related to the excursion measure away from 0 of the reflected process. Note that most of results of this paper have already appeared in Alili and Chaumont (1999).

\section{Fluctuation theory in continuous time}

We start by recalling the fundamental results of fluctuation theory that will be needed in later results. Roughly speaking, fluctuation theory is the study of the process $\left(X_{t}, \bar{X}_{t}, G_{t}\right), t \geqslant 0$, where $G_{t}$ stands for the last time before $t$ at which the past maximum was reached, that is, $G_{t}=\sup \left\{s \leqslant t: X_{s}=\bar{X}_{s}\right\}$. This theory first appeared for random walks in the late $1950 \mathrm{~s}$ and 1960s, (see, for example, Spitzer 1956; Feller 1971; Wendel 1960; Port 1963, and was then developed for continuous time by Rogozin (1966), (see also Fristedt 1974). The following fundamental result from fluctuation theory specifies the joint double Laplace transform of $G$ and $\bar{X}$ taken at an independent exponential time. It is due to Spitzer (1956) for random walks, and to Rogozin (1966) for Lévy processes. We also refer to Wendel (1960) and Greenwood and J.W. Pitman (1980), where an elegant proof based on excursion theory is provided.

Theorem 1 (Spitzer and Rogozin). Let $e$ be an exponential random variable with parameter $\lambda>0$, independent of $X$. Then the following assertions hold:

(i) The couples $\left(G_{e}, \bar{X}_{e}\right)$ and $\left(e-G_{e}, \bar{X}_{e}-X_{e}\right)$ are independent, and both variables $\bar{X}_{e}$ and $\bar{X}_{e}-X_{e}$ have infinitely divisible laws.

(ii) For any $\alpha, \beta \geqslant 0$,

$$
\mathrm{E}\left[\exp \left(-\alpha G_{e}-\beta \bar{X}_{e}\right)\right]=\exp \left(\int_{0}^{\infty} \mathrm{d} t \int_{[0, \infty)}\left(\mathrm{e}^{-\alpha t-\beta x}-1\right) t^{-1} \mathrm{e}^{-\lambda t} P\left(X_{t} \in \mathrm{d} x\right)\right),
$$

and

$$
\mathrm{E}\left[\exp \left(-\alpha\left(e-G_{e}\right)-\beta\left(\bar{X}_{e}-X_{e}\right)\right)\right]=\exp \left(\int_{0}^{\infty} \mathrm{d} t \int_{(-\infty, 0)}\left(\mathrm{e}^{-\alpha t+\beta x}-1\right) t^{-1} \mathrm{e}^{-\lambda t} P\left(X_{t} \in \mathrm{d} x\right)\right) .
$$

Denote by $\psi$ the characteristic exponent of $X$, which is given by

$$
\mathrm{E}\left[\mathrm{e}^{\mathrm{i} x X_{t}}\right]=\mathrm{e}^{-t \psi(x)}, \quad x \in \mathbb{R},
$$

and let us introduce the characteristic functions of $\bar{X}_{e}$ and $-\left(\bar{X}_{e}-X_{e}\right)$, denoted respectively by $\varphi_{\lambda}^{+}$and $\varphi_{\lambda}^{-}$. The so-called Wiener-Hopf factorization identity is the decomposition

$$
\frac{\lambda}{\lambda+\psi}=\varphi_{\lambda}^{+} \varphi_{\lambda}^{-},
$$


which is a straightforward consequence of Theorem 1(i). Another motivation of fluctuation theory, closely related to the process $(G, \bar{X})$, is the study of the law of ladder process associated with $X$. It was proved by Fristedt (1974) that the process $(\tau, H)$ is a bivariate subordinator. So we have

$$
\mathrm{E}\left[\exp \left(-\alpha \tau_{t}-\beta H_{t}\right)\right]=\mathrm{e}^{-t \kappa(\alpha, \beta)}, \quad \alpha, \beta \geqslant 0,
$$

where $\kappa(\cdot, \cdot)$ is the corresponding characteristic exponent. This is specified in the following:

Theorem 2 (Fristedt). For every $\alpha, \beta \geqslant 0$,

$$
\kappa(\alpha, \beta)=k \exp \left(\int_{0}^{\infty} \mathrm{d} t \int_{[0, \infty)}\left(\mathrm{e}^{-t}-\mathrm{e}^{-\alpha t-\beta x}\right) t^{-1} P\left(X_{t} \in \mathrm{d} x\right)\right),
$$

where $k$ is a constant which only depends on the normalization of the local time L.

Now, as a consequence of (2.1) and (2.5), the processes $(\tau, H)$ and $(G, \bar{X})$ are related by the formula:

$$
\mathrm{E}\left[\exp \left(-\alpha G_{e}-\beta \bar{X}_{e}\right)\right]=\frac{\kappa(\lambda, 0)}{\kappa(\alpha+\lambda, \beta)} .
$$

Denote by $\hat{\kappa}$ the characteristic exponent associated with the ladder process of the dual Lévy process $-X$. Then from (2.5) we see that

$$
\begin{aligned}
\kappa(\alpha, 0) \hat{\kappa}(\alpha, 0) & =k \hat{k} \exp \left(\int_{0}^{\infty}\left(\mathrm{e}^{-t}-\mathrm{e}^{-\alpha t}\right) t^{-1} \mathrm{~d} t\right) \\
& =k^{\prime} \alpha,
\end{aligned}
$$

where $k^{\prime}$ and $\hat{k}$ are positive reals. Now, by gathering (2.3), (2.6) and (2.7) we see that the law of $X$ is characterized by those of the ladder heights $H$ and $\hat{H}$. More precisely, we have

$$
k^{\prime} \psi(x)=\kappa(0,-\mathrm{i} x) \hat{\kappa}(0, \mathrm{i} x), \quad x \in \mathbb{R} .
$$

For updated proofs of the Spitzer-Rogozin theorem, Fristedt's theorem, and the above other fluctuation identities above, see Bertoin (1996, Chapter VI) and the references therein.

\section{The fundamental identity.}

For a fixed $x>0$, we denote by $\sigma_{x}$ the time the Lévy process $X$ first hits $(x, \infty)$, that is, $\sigma_{x}=\inf \left\{t: X_{t}>x\right\}$. It is known - see, for instance, the proof of Theorem VI.19 in Bertoin $(1996$, p. 175$)$ - that the process $\left(L_{\sigma_{x}}, x \geqslant 0\right)$ is the right-continuous inverse of $\left(H_{u}, u \geqslant 0\right)$. This shows up in the following theorem, which is a refinement of (1.1). 
Theorem 3. On $(0, \infty)^{3}$, we have the following identity between measures:

$$
t^{-1} P\left(X_{t} \in \mathrm{d} x, L_{\sigma_{x}} \in \mathrm{d} u\right) \mathrm{d} t=u^{-1} P\left(\tau_{u} \in \mathrm{d} t, H_{u} \in \mathrm{d} x\right) \mathrm{d} u .
$$

Proof. This mimics the analytical proof of Theorem 1 in Alili and Doney (1999). In order to simplify the notation, we set

$$
I^{\beta}(\alpha, u)=\mathrm{E}\left[\tau_{u} \mathrm{e}^{-\alpha \tau_{u}-\beta H_{u}}\right] .
$$

To start with, we differentiate (2.5) with respect to $\alpha$ in order to obtain

$$
\begin{aligned}
I^{\beta}(\alpha, u) & =-u\left(\frac{\partial}{\partial \alpha} \kappa(\alpha, \beta)\right) \mathrm{E}\left[\mathrm{e}^{-\alpha \tau_{u}-\beta H_{u}}\right] \\
& =-u\left(\frac{\partial}{\partial u} \mathrm{E}\left[\mathrm{e}^{-\alpha \tau_{u}-\beta H_{u}}\right]\right) \int_{0}^{\infty} \mathrm{d} t \mathrm{e}^{-\alpha t} \mathrm{E}\left[\mathrm{e}^{-\beta X_{t}}\left\{X_{t}>0\right\}\right] \\
& =-u \frac{\partial}{\partial u} \mathrm{E}\left[\int_{0}^{\infty} \mathrm{d} t \exp \left(-\alpha\left(t+\tau_{u}\right)-\beta\left(\tilde{X}_{t}+H_{u}\right)\right) \rrbracket_{\left\{\tilde{X}_{t}>0\right\}}\right],
\end{aligned}
$$

where $\tilde{X}$ is an auxiliary independent copy of the Lévy process $X$. We then perform the change of variables $s=t+\tau_{u}$ and apply Fubini's theorem to obtain

$$
\begin{aligned}
I^{\beta}(\alpha, u) & =-u \frac{\partial}{\partial u} \int_{0}^{\infty} \mathrm{d} s \mathrm{e}^{-\alpha s} \mathrm{E}\left[\mathrm{e}^{-\beta\left(\tilde{X}_{s-\tau_{u}}+H_{u}\right)_{\llbracket}}{\left\{\begin{array}{r}
\left.\tilde{X}_{s-\tau_{u}}>0, \tau_{u} \leqslant s\right\} \\
\end{array}\right]}=-u \frac{\partial}{\partial u} \int_{0}^{\infty} \mathrm{d} s \mathrm{e}^{-\alpha s} \mathrm{E}\left[\mathrm{e}^{-\beta X_{s}\left\{_{\left\{X_{s}>H_{u, \tau_{u}} \leqslant s\right\}}\right],}\right.\right.
\end{aligned}
$$

where the latter equality is obtained by the strong Markov property. We now condition on $X_{s}$ and use the fact that $\left(L_{\sigma_{x}}, x \geqslant 0\right)$ is the right-continuous inverse of $\left(H_{u}, u \geqslant 0\right)$. By replacing the event $\left\{X_{s}>H_{u}\right\}$ by $\left\{L_{\sigma_{X_{s}}}>u, X_{s} \geqslant 0\right\}$, we finally find that

$$
I^{\beta}(\alpha, u)=-u \frac{\partial}{\partial u} \int_{0}^{\infty} \mathrm{d} s \mathrm{e}^{-\alpha s} \int_{(0, \infty)} \mathrm{e}^{-\beta z} P\left(X_{s} \in \mathrm{d} z, L_{\sigma\left(X_{s}\right)}>u, X_{s} \geqslant 0\right)
$$

and the result follows by the injectivity of the double Laplace transform (in $\alpha$ and $\beta$ ).

Remark 1. The difficulty in using the path transform method of Alili and Doney (1999) and Marchal (2000) relies on the fact that for Lévy processes there are unaccountably many ladder indices. It would be interesting to find a path-transformation proof of Theorem 3, following these authors.

The following result is an equivalent form of Theorem 3. Here we express the Laplace transform of the process $\left(\left(L_{\sigma\left(X_{t}\right)}, X_{t}\right), t \geqslant 0\right)$ in terms of the Laplace exponent of $(\tau, H)$. This then leads to an expression of the conditional law of $L_{\sigma\left(X_{e}\right)}$ given $X_{e}=x$, where $e$ is an independent exponential time. 
Theorem 4. Let $e$ be an independent exponential time with parameter $\lambda$ and $\alpha, \beta \geqslant 0$. Then we have

$$
\mathrm{E}\left[\exp \left(-\alpha L_{\sigma\left(X_{e}\right)}-\beta X_{e}\right) \mid X_{e} \geqslant 0\right]=\frac{\kappa(\lambda, 0)}{\frac{\partial}{\partial \lambda} \kappa(\lambda, 0)} \frac{\frac{\partial}{\partial \lambda} \kappa(\lambda, \beta)}{\alpha+\kappa(\lambda, \beta)}
$$

Consequently, conditionally on $X_{e} \geqslant 0$, the variable $L_{\sigma\left(X_{e}\right)}$ is exponentially distributed with parameter $\kappa(\lambda, 0)$. Moreover, the law of $X_{e}$ conditionally on $X_{e} \geqslant 0$ and $L_{\sigma\left(X_{e}\right)}=x$ is given by

$$
\mathrm{E}\left[\mathrm{e}^{-\beta X_{e}} \mid X_{e} \geqslant 0, L_{\sigma\left(X_{e}\right)}=x\right]=\frac{\frac{\partial}{\partial \lambda} \kappa(\lambda, \beta)}{\frac{\partial}{\partial \lambda} \kappa(\lambda, 0)} \mathrm{e}^{-(\kappa(\lambda, \beta)-\kappa(\lambda, 0)) x}, \quad \beta \geqslant 0 .
$$

Proof. Theorem 3 implies that

$$
\begin{aligned}
\mathrm{E}\left[\exp \left(-\alpha L_{\sigma\left(X_{\mathrm{e}}\right)}-\beta X_{e}\right) \rrbracket_{\left\{X_{e} \geqslant 0\right\}}\right] & =\lambda \int_{0}^{\infty} \int_{0}^{\infty} t \mathrm{~d} t u^{-1} \mathrm{~d} u \mathrm{e}^{-\lambda t-\alpha u} \mathrm{E}\left[\tau_{u} \in \mathrm{d} t, \mathrm{e}^{-\beta H_{u}}\right] \\
& =\lambda \int_{0}^{\infty} u^{-1} \mathrm{e}^{-\alpha u} \mathrm{E}\left[\tau_{u} \mathrm{e}^{-\lambda \tau_{u}-\beta H_{u}}\right] \mathrm{d} u .
\end{aligned}
$$

Differentiating with respect to $\alpha$, it follows that

$$
\begin{aligned}
\left.\mathrm{E}\left[L_{\sigma\left(X_{e}\right)} \exp \left(-\alpha L_{\sigma\left(X_{e}\right)}-\beta X_{e}\right)\right\}_{\left\{X_{e} \geqslant 0\right\}}\right] & =\lambda \int_{0}^{\infty} \mathrm{d} u \mathrm{e}^{-\alpha u} \mathrm{E}\left[\tau_{u} \mathrm{e}^{-\lambda \tau_{u}-\beta H_{u}}\right] \\
& =-\lambda \frac{\partial}{\partial \lambda} \int_{0}^{\infty} \mathrm{d} u \mathrm{e}^{-\alpha u} \mathrm{E}\left[\mathrm{e}^{-\lambda \tau_{u}-\beta H_{u}}\right] \\
& =(\alpha+\kappa(\lambda, \beta))^{-2} \lambda \frac{\partial}{\partial \lambda} \kappa(\lambda, \beta) .
\end{aligned}
$$

We conclude by integrating with respect to $\alpha$. The second statement of the theorem is a straight consequence of Theorem 4 , and the last one follows from

$$
\int_{0}^{\infty} \mathrm{e}^{-(\alpha+\kappa(\lambda, 0)) x} \mathrm{E}\left[\mathrm{e}^{-\beta X_{e}} \mid X_{e} \geqslant 0, L_{\sigma\left(X_{e}\right)}=x\right] \mathrm{d} x=\frac{1}{\frac{\partial}{\partial \lambda} \kappa(\lambda, 0)} \frac{\frac{\partial}{\partial \lambda} \kappa(\lambda, \beta)}{\alpha+\kappa(\lambda, \beta)} .
$$

When $X$ has no negative jumps, $\bar{X}$ is a continuous increasing additive functional of the reflected process $\bar{X}-X$. Moreover, the set of its increase times corresponds to the zero set of $\bar{X}-X$, which means that $\bar{X}$ is a local time at zero of $\bar{X}-X$. Since the local time is 
unique up to a multiplicative constant, we can fix its normalization such that $L=\bar{X}$. Then the variable $L_{\sigma\left(X_{e}\right)}$ involved in the above theorem is almost surely equal $X_{e}$. On the other hand, it is known that $\bar{X}_{e}$ is exponentially distributed with parameter $\kappa(\lambda, 0)$; see, for instance, Corollary VII.2 in Bertoin (1996, p. 190). We then obtain from Theorem 4 that

$$
P\left(\bar{X}_{e} \in \mathrm{d} x\right)=P\left(X_{e} \in \mathrm{d} x \mid X_{e} \geqslant 0\right), \quad x \geqslant 0,
$$

each member being exponentially distributed with parameter $\kappa(\lambda, 0)$. In the general case, it is not difficult to check that for every $t>0$ the equalities $L_{\sigma\left(\bar{X}_{t}\right)}=L_{G_{t}}=L_{t}$ hold almost surely. Moreover, it is well known from excursion theory that $L_{e}$ is exponentially distributed with parameter $\kappa(\lambda, 0)$. Consequently, identity (3.6) might be generalized as follows:

Corollary 1. Let e be as in Theorem 4. Then

$$
P\left(L_{\sigma\left(\bar{X}_{e}\right)} \in \mathrm{d} x\right)=P\left(L_{\sigma\left(X_{e}\right)} \in \mathrm{d} x \mid X_{e} \geqslant 0\right), \quad x \geqslant 0 .
$$

Note also that the identities (3.6) and (3.7) hold at fixed time whenever $P\left(X_{t} \geqslant 0\right)$ does not depend on $t$. Identity (3.6) was proved in the stable case by Skorohod (1971); see also Bingham (1975).

\section{The stable case}

In this section we suppose that $X$ is a stable Lévy process with index $\alpha \in(0,2]$; that is, for every $s>0, X$ satisfies the scaling property

$$
\left(X_{t}, t \geqslant 0\right) \stackrel{d}{=}\left(s^{-1} X_{s^{a} t}, t \geqslant 0\right) .
$$

We then see that the ladder process $(\tau, H)$ is stable with index $(\rho, \alpha \rho)$. More precisely, from Theorem 2, we easily deduce that

$$
\left(\tau_{t}, H_{t}, t \geqslant 0\right) \stackrel{d}{=}\left(s^{-1 / \rho} \tau_{s t}, s^{-1 / \alpha \rho} H_{s t}, t \geqslant 0\right),
$$

where $\rho$ is given by $\rho=P\left(X_{1} \geqslant 0\right)$. This allows us to translate Theorem 3 into the following identity in law:

Corollary 2. The couple $\left(L_{\sigma\left(X_{1}\right)}, X_{1}\right)$ conditionally on $X_{1} \geqslant 0$ and $\left(\tau_{1}^{-\rho}, \tau_{1}^{-1 / \alpha} H_{1}\right)$ are identically distributed.

Note that by the scaling property of $X$, the bivariate process $\left(L_{\sigma\left(X_{t}\right)}, X_{t}, t \geqslant 0\right)$ is also stable with index $\left(\rho^{-1}, \alpha\right)$. Therefore, the above corollary can be extended to any fixed time $t \geqslant 0$. 
Proof. On the one hand, we have for every $\lambda>0$, and $\theta, \beta \geqslant 0$,

$$
\begin{aligned}
& \int_{0}^{\infty} \mathrm{e}^{-\lambda u} \mathrm{E}\left[\exp \left(-\theta \tau_{u}-\beta H_{u}\right)\right] \mathrm{d} u=\int_{0}^{\infty} \mathrm{e}^{-\lambda u} \mathrm{E}\left[\exp \left(-\theta u^{1 / \rho} \tau_{1}-\beta u^{1 / \alpha \rho} H_{1}\right)\right] \mathrm{d} u \\
& =\rho \int_{0}^{\infty} \mathrm{e}^{-\theta t} \mathrm{E}\left[\left(\frac{t}{\tau_{1}}\right)^{\rho} \exp \left(-\lambda\left(\frac{t}{\tau_{1}}\right)^{\rho}-\beta\left(\frac{t}{\tau_{1}}\right)^{1 / \alpha} H_{1}\right)\right] \frac{\mathrm{d} t}{t},
\end{aligned}
$$

where the first equality comes from the scaling property, and the second from the change of variables $u=\left(t / \tau_{1}\right)^{\rho}$. On the other hand, by applying Theorem 3, we see that

$$
\int_{0}^{\infty} \mathrm{e}^{-\lambda u} \mathrm{E}\left[\exp -\theta \tau_{u}-\beta H_{u}\right] \mathrm{d} u=\int_{0}^{\infty} \mathrm{e}^{-\theta t} \mathrm{E}\left[L_{\sigma\left(X_{t}\right)} \exp \left(-\lambda L_{\sigma\left(X_{t}\right)}-\beta X_{t}\right)_{\left\{X_{t}>0\right\}}\right] \frac{\mathrm{d} t}{t} .
$$

Integrating these identities with respect to $\lambda$ and using the scaling property of the process $\left(L_{\sigma\left(X_{t}\right)}, X_{t}, t \geqslant 0\right)$ we obtain

$$
\begin{aligned}
& \rho \int_{0}^{\infty} \mathrm{e}^{-\theta t} \mathrm{E}\left[\exp \left(-\lambda\left(\frac{t}{\tau_{1}}\right)^{\rho}-\beta\left(\frac{t}{\tau_{1}}\right)^{1 / \alpha} H_{1}\right)\right] \frac{\mathrm{d} t}{t} \\
& \left.\quad=\int_{0}^{\infty} \mathrm{e}^{-\theta t} \mathrm{E}\left[\exp \left(-\lambda t^{\rho} L_{\sigma\left(X_{1}\right)}-\beta t^{1 / \alpha} X_{1}\right)\right]_{\left\{X_{1}>0\right\}}\right] \frac{\mathrm{d} t}{t} \\
& \quad=\rho \int_{0}^{\infty} \mathrm{e}^{-\theta t} \mathrm{E}\left[\exp \left(-\lambda t^{\rho} L_{\sigma\left(X_{1}\right)}-\beta t^{1 / \alpha} X_{1}\right) \mid X_{1} \geqslant 0\right] \frac{\mathrm{d} t}{t},
\end{aligned}
$$

which is the required result.

As well as for random walks, the renewal measure associated with the ladder process $(\tau, H)$ plays an important role in the study of the Martin boundaries associated with a killed Lévy process; see Alili and Doney, (2000) for a recent study of the discrete setting. In the following some results will be expressed in terms of this measure on $\mathbb{R}^{+} \times \mathbb{R}^{+}$for which we recall the definition:

$$
g(\mathrm{~d} t, \mathrm{~d} x)=\int_{0}^{\infty} P\left(\tau_{u} \in \mathrm{d} t, H_{u} \in \mathrm{d} x\right) \mathrm{d} u .
$$

Differentiating (4.8) and following the same reasoning as in the proof of the fundamental identity (Theorem 3), we obtain the following convolution equation which will be useful later:

$$
x g(\mathrm{~d} t, \mathrm{~d} x)=\mathrm{d} t \int_{u=0}^{t} \int_{z=0}^{x} \frac{x-z}{t-u} P_{z}\left(X_{t-u} \in \mathrm{d} x\right) g(\mathrm{~d} u, \mathrm{~d} z), \quad x>0 .
$$

We refer to Theorem 1 in Alili and Doney (1999) for a discrete-time version of this identity.

Recall that $G_{t}$, defined in Section 2, is the last time at which $X$ reaches its absolute maximum before $t$. It is well known (see Theorem VI.3.13 in Bertoin 1996) that the law of $t^{-1} G_{t}$ is the generalized arcsine law with parameter $\rho$ : 


$$
P\left(t^{-1} G_{t} \in \mathrm{d} s\right)=\frac{\sin \rho \pi}{\pi} s^{\rho-1}(1-s)^{-\rho} \mathrm{d} s, \quad 0<s<1 .
$$

Let $P\left(\bar{X}_{t} \in \mathrm{d} x \mid G_{t}=s\right)$ be a regular version, in $s$, of the conditional law of $\bar{X}_{t}$ given $G_{t}$. The law of the couple $\left(\bar{X}_{t}, G_{t}\right)$ can be characterized in terms of $g$ in the following way.

Corollary 3. For every $s$ and $t$ such that $0<s<t$ and $x>0$, the following identities between measures hold:

$$
\begin{aligned}
\Gamma(1-\rho) s^{\rho} P\left(\bar{X}_{t} \in \mathrm{d} x \mid G_{t}=s\right) \mathrm{d} s & =\mathrm{E}\left[L_{\sigma\left(X_{s}\right)}, X_{s} \in \mathrm{d} x\right] \mathrm{d} s \\
& =s g(\mathrm{~d} s, \mathrm{~d} x) .
\end{aligned}
$$

We emphasize that the left-hand side does not depend on $t$.

Proof. Starting from (2.6), we see that for all positive reals, $\gamma, \beta$ and $\lambda$,

$$
\begin{aligned}
\int_{0}^{\infty} & \mathrm{e}^{-\lambda t} \mathrm{E}\left[\exp \left(\gamma G_{t}-\beta \bar{X}_{t}\right)\right] \mathrm{d} t \\
& =\lambda^{-1} \kappa(\lambda, 0) \int_{0}^{\infty} \mathrm{E}\left[\exp \left(-(\gamma+\lambda) \tau_{u}-\beta H_{u}\right)\right] \mathrm{d} u \\
& =\Gamma(1-\rho)^{-1} \mathrm{E}\left[\int_{0}^{\infty} \int_{0}^{\infty} x^{-\rho} \exp \left(-\lambda\left(x+\tau_{u}\right)\right) \exp \left(-\gamma \tau_{u}-\beta H_{u}\right) \mathrm{d} u \mathrm{~d} x\right] \\
& =\Gamma(1-\rho)^{-1} \mathrm{E}\left[\int_{0}^{\infty} \int_{\tau_{u}}^{\infty} \mathrm{e}^{-\lambda t}\left(t-\tau_{u}\right)^{-\rho} \exp \left(-\gamma \tau_{u}-\beta H_{u}\right) \mathrm{d} u \mathrm{~d} x\right],
\end{aligned}
$$

where the last equality is obtained by performing the change of variable $t=x+\tau_{u}$. Inverting the Laplace transform in $\lambda$ and using the scaling property of $(\tau, H)$, we obtain

$$
\begin{aligned}
& \mathrm{E}\left[\exp \left(-\gamma G_{t}-\beta \bar{X}_{t}\right)\right] \\
& =\Gamma(1-\rho)^{-1} \int_{0}^{\infty} \mathrm{E}\left[\left(t-\tau_{u}\right)^{-\rho} \exp \left(-\gamma \tau_{u}-\beta H_{u}\right)^{\complement}\left\{_{\left\{\tau_{u} \leqslant t\right\}}\right] \mathrm{d} u\right. \\
& \left.=\Gamma(1-\rho)^{-1} \int_{0}^{\infty} \mathrm{E}\left[\left(t-u^{1 / \rho} \tau_{1}\right)^{-\rho} \exp \left(-\gamma u^{1 / \rho} \tau_{1}-\beta u^{1 / \alpha \rho} H_{1}\right)\right]_{\left\{u^{1 / \rho} \tau_{1} \leqslant t\right\}}\right] \mathrm{d} u .
\end{aligned}
$$

By the change of variables $u^{1 / \rho} \tau_{1}=z$, we have

$$
\mathrm{E}\left[\exp \left(-\gamma G_{t}-\beta \bar{X}_{t}\right)\right]=\rho \Gamma(1-\rho)^{-1} \int_{0}^{t} z^{\rho-1}(t-z)^{-\rho} \mathrm{e}^{-\gamma z} \mathrm{E}\left[\tau_{1}^{-\rho} \exp \left(-\beta\left(\frac{z}{\tau_{1}}\right)^{1 / \alpha} H_{1}\right)\right] \mathrm{d} z,
$$

which shows that

$$
\mathrm{E}\left[\mathrm{e}^{-\beta S_{t}} \mid G_{t}=z\right]=\rho \Gamma(1-\rho)^{-1} \mathrm{E}\left[\tau_{1}^{-\rho} \exp \left(-\beta\left(\frac{z}{\tau_{1}}\right)^{1 / \alpha} H_{1}\right)\right] .
$$


We then obtain the result by using Corollary 2 .

We point out that Corollary 3 provides a better understanding of the exit law of the socalled stable meander for which we recall the definition. Let $g_{t}$ be the first time before $t$ at which $X$ reaches its past overall minimum,

$$
g_{t} \stackrel{\text { def }}{=} \inf \left\{u: X_{u}=\underline{X}_{t}\right\},
$$

where $\underline{X}_{t} \stackrel{\text { def }}{=} \inf _{u \leqslant t} X_{u}$. The meander $m$ of length $s$, with $s<t$, is a process defined on the time interval $[0, s]$ with the same law as the process $\left\{(X-\underline{X})_{g_{t}+u}, 0 \leqslant u \leqslant t-g_{t}\right\}$ conditionally on $t-g_{t}=s$. We refer to Chaumont (1997) for a more complete definition and study of this process. Note that from the time reversal property of Lévy processes (see Lemma 2 in Bertoin 1996, Section II.1), we have

$$
P\left(\bar{X}_{t} \in \mathrm{d} x \mid G_{t}=s\right)=P\left((X-\underline{X})_{t} \in \mathrm{d} x \mid g_{t}=s\right) .
$$

We may now reformulate the identities (4.10) and (4.11) in terms of the meander's exit law.

Corollary 4. With the above notation, the exit law of the stable meander m, with length $s$, is specified by

$$
P\left(m_{s} \in \mathrm{d} x\right) \mathrm{d} s=\Gamma(1-\rho)^{-1} s^{1-\rho} g(\mathrm{~d} s, \mathrm{~d} x) .
$$

\section{Application to the excursion measure of the reflected process}

Consider the process $X$ reflected by its past minimum, which is $X-\underline{X}$, where $\underline{X}_{t} \stackrel{\text { def }}{=} \inf _{s \leqslant t} X_{s}$, for each $t \geqslant 0$. Note that $X-\underline{X}=\overline{\hat{X}}-\hat{X}$, where $\hat{X}=-X$, so by duality this process enjoys all the properties of $\bar{X}-X$, described in the Introduction. In particular, it is strongly Markovian and, under our hypothesis, 0 is regular for itself. It then makes sense to introduce the Itô measure $\underline{n}$ of its excursions away from 0 . In this section we are interested in the description of the entrance law under $\underline{n}$. This measure fulfils the strong Markov property in a sense which is made precise in (5.3). So, the law of the excursion process $\left(e_{t}\right)_{t \geqslant 0}$ under $\underline{n}$ is entirely determined by both its semigroup and its entrance law. The semigroup under $\underline{n}$ is well known and is defined as follows. For fixed positive reals $x$ and $t$, we denote by $p_{t}^{0}(x, \mathrm{~d} y)$, $y \geqslant 0$, the semigroup of the process $X$ killed when it hits the negative half-line $(-\infty, 0)$. That is, for any bounded or non-negative Borel function $f$,

$$
\mathrm{E}_{x}\left[f\left(X_{t}\right)_{\{t<\tau\}}\right] \stackrel{\text { def }}{=} \int_{0}^{\infty} f(y) p_{t}^{0}(x, \mathrm{~d} y),
$$

where $\tau \stackrel{\text { def }}{=} \inf \left\{t: X_{t}<0\right\}$. The semigroup $p_{t}^{0}(x, \mathrm{~d} y)$ is the same as that of the excursion process $\left(e_{t}\right)_{t \geqslant 0}$ under $\underline{n}$. The entrance law, $\left(q_{t}(\mathrm{~d} x)\right)_{t>0}, x>0$, under $\underline{n}$ is defined, for any bounded measurable function $f$, by

$$
\underline{n}\left(f\left(e_{t}\right), t<\zeta\right)=\int_{0}^{\infty} f(x) q_{t}(\mathrm{~d} x),
$$


where $\zeta$ is the lifetime of the generic excursion. With this notation, the measure $\underline{n}$ can be described as follows: for every $0<t_{1}<\ldots<t_{n}<\infty$ and bounded Borel functions $f_{1}, \ldots, f_{n}$,

$$
\begin{aligned}
& \underline{n}\left(f_{1}\left(e_{t_{1}}\right)\right) \ldots f_{n}\left(e_{t_{n}}\right)\left\{_{\left\{t_{n}<\xi\right\}}\right) \\
& =\int_{\mathbb{R}^{n}} f_{1}\left(x_{1}\right) \ldots f_{n}\left(x_{n}\right) q_{t_{1}}\left(\mathrm{~d} x_{1}\right) p_{t_{2}-t_{1}}^{0}\left(x_{1}, \mathrm{~d} x_{2}\right) \ldots p_{t_{n}-t_{n-1}}^{0}\left(x_{n-1}, \mathrm{~d} x_{n}\right) .
\end{aligned}
$$

For a complete description of $\underline{n}$, it remains to note that under this measure, the generic excursion starts from 0 at time $t=0$.

In general, the entrance law $\left(q_{t}\right)_{t>0}$ under $\underline{n}$ is much less known than the semigroup $\left(p_{t}^{0}\right)_{t \geqslant 0}$ described above. However, when $X$ has no positive jumps, we know from Bertoin (1996, Section VII.3) that

$$
q_{t}(\mathrm{~d} x)=k t^{-1} x p_{t}(\mathrm{~d} x), \quad x>0,
$$

where $\left(p_{t}\right)_{t>0}$ is the entrance law of $X$ and $k$ is a constant independent of $t$ and $x$. In the following proposition, we show how the results of Section 3 allows us to extend relation (5.4) to all Lévy processes considered here.

Theorem 5. For every $t>0$, we have

$$
\begin{aligned}
q_{t}(\mathrm{~d} x) \mathrm{d} t & =k^{\prime \prime} t^{-1} \mathrm{E}\left[L_{\sigma_{x}}, X_{t} \in \mathrm{d} x\right] \mathrm{d} t, \quad x>0, \\
& =k g(\mathrm{~d} t, \mathrm{~d} x),
\end{aligned}
$$

where $k^{\prime \prime}$ is a constant independent of $t$ and $x$, and $g$ is the renewal measure defined in (4.8).

Proof. From the exit formula of excursion theory, (see Bertoin 1996, Section IV.4), we obtain

$$
\underline{n}\left(\int_{0}^{\zeta} \mathrm{e}^{-\alpha t-\beta e_{t}} \mathrm{~d} t\right)=\hat{\kappa}(\alpha, 0) \mathrm{E}\left[\int_{0}^{\infty} \mathrm{e}^{\left.-\alpha t-\beta(X-\underline{X})_{t} \mathrm{~d} t\right] .}\right.
$$

Applying the Spitzer-Rogozin identity (Theorem 1), we see that

$$
\underline{n}\left(\int_{0}^{\xi} \mathrm{e}^{-\alpha t-\beta e_{t}} \mathrm{~d} t\right)=\frac{\hat{\kappa}(\alpha, 0)}{\alpha} \exp \left(\int_{0}^{\infty} \mathrm{d} t \int_{0}^{\infty}\left(\mathrm{e}^{-\beta x}-1\right) \frac{\mathrm{e}^{-\alpha t}}{t} P\left(X_{t} \in \mathrm{d} x\right)\right) .
$$

On the other hand, from Fristedt's identity (Theorem 2) and (2.7), we obtain

$$
\begin{aligned}
& \int_{0}^{\infty} \int_{0}^{\infty} \mathrm{e}^{-\alpha t-\beta x} \int_{0}^{\infty} P\left(\tau_{u} \in \mathrm{d} t, X_{\tau_{u}} \in \mathrm{d} x\right) \mathrm{d} u=(\kappa(\alpha, \beta))^{-1} \\
& =k^{-1} \exp \left(\int_{0}^{\infty} \mathrm{d} t \int_{0}^{\infty}\left(\mathrm{e}^{-\alpha t-\beta x}-\mathrm{e}^{-t}\right) \frac{1}{t} P\left(X_{t} \in \mathrm{d} x\right)\right) \\
& \quad=k^{-1} \frac{\hat{\kappa}(\alpha, 0)}{\alpha} \exp \left(\int_{0}^{\infty} \mathrm{d} t \int_{0}^{\infty}\left(\mathrm{e}^{-\beta x}-1\right) \frac{\mathrm{e}^{-\alpha t}}{t} P\left(X_{t} \in \mathrm{d} x\right)\right) .
\end{aligned}
$$


From (5.6) and (5.7) and using Theorem 3, we finaly obtain

$$
\begin{aligned}
\underline{n}\left(e_{t} \in \mathrm{d} x, t<\zeta\right) \mathrm{d} t & =k^{\prime \prime} \int_{0}^{\infty} P\left(\tau_{u} \in \mathrm{d} t, X_{\tau_{u}} \in \mathrm{d} x\right) \mathrm{d} u \\
& =\frac{k^{\prime \prime}}{t} \mathrm{E}\left[L_{\sigma_{x}}, X_{t} \in \mathrm{d} x\right] \mathrm{d} t .
\end{aligned}
$$

The above result is also motivated by the connection between $q_{t}$ and the entrance law of the Lévy process conditioned to stay positive, which we now introduce. To this end, we first make two additional assumptions. Suppose that, for each $t>0$, the semigroup $P_{t}$, defined for every bounded Borel function $f$ by

$$
P_{t} f(x)=\mathrm{E}_{x}\left[f\left(X_{t}\right)\right]=\mathrm{E}\left[f\left(X_{t}+x\right)\right], \quad x \in \mathbb{R},
$$

satisfies the strong Feller property. This property is equivalent to the absolute continuity of the law of $X_{t}$ with respect to the Lebesgue measure for any fixed $t>0$; see, for instance, Bertoin (1996). We also suppose that

$$
\lim _{t \rightarrow \infty} \bar{X}_{t}=+\infty .
$$

Under these additional hypothesis we can introduce the Lévy process conditioned to stay positive $X^{\uparrow, x}, x>0$, as follows (for more details, see Chaumont 1996). Denote by $\hat{L}$ the local time at 0 of the process $X-\underline{X}$; and for any positive $x$, let $\hat{\sigma}_{x}$ be the first entrance time in $(x, \infty)$, by $\hat{X}=-X$, that is, $\hat{\sigma}_{x}=\inf \left\{t: \hat{X}_{t}>x\right\}$. Now the function $\hat{h}$ defined by

$$
\hat{h}(x) \stackrel{\text { def }}{=} \mathrm{E}\left[\hat{L}_{\hat{\sigma}_{x}}\right], \quad x>0,
$$

is a positive harmonic function for the semigroup $p_{t}^{0}(x, \mathrm{~d} y)$ and $X^{\uparrow, x}, x>0$, is the $h$-process in Doob's sense defined with respect to $\hat{h}$ and $p_{t}^{0}(x, y)$. More formally, if $p_{t}^{\uparrow}(x, y), x, y>0$, denotes the semigroup of the process $X^{\uparrow, x}, x>0$, then we have

$$
p_{t}^{\uparrow}(x, \mathrm{~d} y)=\frac{\hat{h}(y)}{\hat{h}(x)} p_{t}^{0}(x, \mathrm{~d} y), \quad t, x, y>0 .
$$

Obviously, for every $x>0, X^{\uparrow, x}$ is a positive strong Markov process starting from $x$. Moreover, it can easily be proved that $\lim _{t \rightarrow \infty} X_{t}^{\uparrow, x}=+\infty$ almost surely. In the Brownian case, note that the latter process corresponds to the three-dimensional Bessel process. Following Chaumont (1996), it is possible to construct a process $X^{\uparrow, 0}$, starting from 0 , whose semigroup in $(0, \infty)$ is precisely $p_{t}^{\uparrow}(x, \mathrm{~d} y)$. It has been shown that, in some cases, the family of processes $X^{\uparrow, x}, x>0$, converges weakly towards the law of $X^{\uparrow, 0}$; see, for instance, Chaumont (1996). From Theorem 3 in Chaumont (1996), the entrance law $p_{t}^{\uparrow}(\mathrm{d} y)$ of $X^{\uparrow, 0}$ is related to $q_{t}(\mathrm{~d} y)$ as follows:

$$
p_{t}^{\uparrow}(\mathrm{d} y)=\hat{h}(y) q_{t}(\mathrm{~d} y) .
$$

So from Theorem 5 and the above identity, we obtain: 
Proposition 1. For every positive $t$ and $x$,

$$
p_{t}^{\uparrow}(x)=k^{\prime \prime} t^{-1} \hat{h}(x) \mathrm{E}\left[L_{\sigma_{x}}, X_{t} \in \mathrm{d} x\right],
$$

where $k^{\prime \prime}$ is a constant independent of $x$ and $t$.

We conclude this paper by stating an open problem. A natural problem is to calculate the conditional expectation

$$
\mathrm{E}\left[L_{\sigma_{x}} \mid X_{t}=x\right]
$$

which has appeared throughout this paper. This is actually equivalent to expressing the renewal measure $g(\mathrm{~d} s, \mathrm{~d} x)$ in terms of the entrance law of $X$, and it allows us to express explicitly the Wiener-Hopf factors specified in (2.3). Theoretically, from Theorem 4, we should be able to answer this question by inverting the Laplace transform of (3.5) in $\lambda$ and $\beta$. In the case where $X$ has no positive jumps, since $L_{\sigma_{x}}$ is deterministic, as we have already observed, the above conditional expectation is identically equal to $x$. This example incites us to formulate the following:

Problem. Identify the class of Lévy processes for which the conditional expectation (5.14) above does not depend on $t$. The latter expression is then obviously equal to the harmonic function $h(x) \stackrel{\text { def }}{=} \mathrm{E}\left[L_{\sigma_{x}}\right]$.

We have checked that this property fails in the case of the symmetric Cauchy process. To see this, recall that the semigroup of $X$ is then given by $P_{x}\left(X_{t} \in \mathrm{d} y\right)=$ $p_{t}(x, \mathrm{~d} y)=\pi^{-1} t\left((x-y)^{2}+t^{2}\right)^{-1} \mathrm{~d} y, \quad x, y \in \mathbb{R}, \quad t \geqslant 0$, and the corresponding renewal function is, up to a constant, given by $h(x)=x^{1 / 2}$. Under the above hypothesis and from Corollary 3 , the renewal measure $g$ would be $g(\mathrm{~d} t, \mathrm{~d} x)=x^{1 / 2} \pi^{-1}\left(x^{2}+t^{2}\right)^{-1} \mathrm{~d} t \mathrm{~d} x$. From (4.9) we would have

$$
\frac{x^{3 / 2}}{x^{2}+t^{2}}=\pi^{-1} \int_{0}^{x} \mathrm{~d} z \int_{0}^{t} \mathrm{~d} u \frac{x-z}{t-u} \frac{t-u}{(x-z)^{2}+(t-u)^{2}} \frac{\sqrt{z}}{z^{2}+u^{2}} .
$$

But one can easily check that

$$
\frac{x^{3 / 2}}{x^{2}+t^{2}}=\pi^{-1} \int_{0}^{x} \mathrm{~d} z \int_{\mathbb{R}} \mathrm{d} u \frac{x-z}{t-u} \frac{t-u}{(x-z)^{2}+(t-u)^{2}} \frac{\sqrt{z}}{z^{2}+u^{2}} .
$$

So equation (5.15) fails.

\section{References}

Alili, L. and Chaumont, L. (1999) Quelques nouvelles identités de fluctuation pour les processus de Lévy. C. R. Acad. Sci. Paris Sér. I. 328, 613-616.

Alili, L. and Doney, R.A. (1999) Wiener-Hopf factorization revisited and some applications. Stochastics Stochastics Rep., 66, 87-102. 
Alili, L. and Doney, R.A. (2000) Martin boundary associated with a killed random walk. Ann. Inst. H. Poincaré Probab. Statist. To appear.

Bertoin, J. (1996) Lévy Processes. Cambridge: Cambridge University Press.

Bertoin, J. and Doney, R.A. (1997) Spitzer's condition for random walks and Lévy processes. Ann. Inst. H. Poincaré Probab. Statist., 33, 167-178.

Bingham, N.H. (1975) Fluctuation theory in continuous time. Adv. Appl. Probab., 7, 705-766.

Chaumont, L. (1996) Conditionings and path decompositions for Lévy processes. Stochastic Process Appl., 64, 39-54.

Chaumont, L. (1997) Excursion normalisée, méandre et pont pour des processus stables. Bull. Sci. Math., 121, 377-403.

Feller, W.E. (1971) An introduction to probability theory and its applications, Vol. 2, 2nd edition. New York: Wiley.

Fristedt, B.E. (1974) Sample functions of stochastic processes with stationary, independent increments. In P. Ney and S. Port (eds), Advances in Probability and Related Topics, Vol. 3, pp. 241-396. New York: Marcel Dekker.

Greenwood P. and Pitman, J.W. (1980) Fluctuation identities for Lévy processes and splitting at the maximum. Adv. Appl. Probab., 12, 893-902.

Marchal, P. (2001) On a new Wiener-Hopf factorization by Alili and Doney. In J. Azéma, M. Émery, M. Ledoux and M. Yor (eds), Séminaire de Probabilités XXXV, Lecture Notes in Math. 1755. Berlin: Springer-Verlag.

Port, S.C. (1963) An elementary approach to fluctuation theory. J. Math. Anal. Appl., 6, 109-151.

Rogozin, B.A. (1966) On the distribution of functionals related to boundary problems for processes with independent increments. Theory Probab. Appl., 11, 580-591.

Spitzer, F. (1956) A combinatorial lemma and its applications to probability theory. Trans. Amer. Math. Soc., 82, 323-339.

Skorohod, A.V. (1971) Theory of Random Processes. Vol. 1. Moscow: Nauka.

Wendel, J.G. (1960) Order statistics of partial sums. Ann. Math. Statist., 31, 1034-1044.

Received April 2000 and revised December 2000 